\title{
THE VOYAGER COSMIC RAY EXPERIMENT
}

D. E. Stilwell, W. D. Davis, R. M. Joyce, F. B. McDonald and J. H. Trainor*

W. E. Althouse, A. C. Cummings, T. L. Garrard, E. C. Stone and R. E. Vogt**

\section{Abstract}

The Voyager Cosmic Ray Experiment includes seven $\mathrm{dE} / \mathrm{dx}-$ $E$ telescopes to measure the energy and charge of particles of $1 \leq \mathrm{Z} \leq 26$ in the energy range $1-500 \mathrm{MeV} /$ nucleon and to measure electron energy in the range $3 \leq \mathrm{E}_{\mathrm{e}}<110 \mathrm{MeV}$. Isotopic composition of hydrogen through sulfur in the range up to $75 \mathrm{MeV} /$ nucleon can also be resolved. The electronic systems include a dual-gain, charge sensitive preamplifier, 4096-channel pulse height analyzers for three parameter analysis of selected events, and an event type readout polling scheme to maximize the use of available telemetry space and to enhance the occurrence of rare events in the data. Details of the detector, electronic and mechanical design are presented.

\section{Introduction}

The Voyager Cosmic Ray Instrument, launched on each of the two Voyager spacecraft in August and September, 1977, will provide measurements of the cosmic ray flux and energy spectra during the cruise phase to Jupiter and Saturn and will provide useful measurements of Jovian trapped radiation during much of the planetary encounter. The instrument contains 46 silicon solidstate detectors arranged in seven telescopes to determine particle charge and energy in the range $1<Z<26$ and $1 \mathrm{MeV}$ to $500 \mathrm{MeV} /$ nucleon. The scientific goals and details of the telescope design parameters have been described by Stone et al. (Ref. 1).

The associated electronics systems detect 45 different coincidence/anticoincidence logic conditions corresponding to various particle types within separate energy windows, and originating from various telescopes. These event rates are counted and telemetered; along with 56 different "singles" rates. Certain events are selected for pulse-height analysis. The system includes 8 PHAs which are shared among 30 detectors. Events analyzed are three-parameter, double $\mathrm{dE} / \mathrm{dx}$ vs. $E$ in form except for the electron measurements. Multiple storage registers for PHA data, each dedicated to specific event types, and a readout polling scheme maximize the efficient use of available telemetry space. Certain charge-sensitive preamplifiers may be operated in either of two gain modes to include a wide range of 2 (particles up to and including iron nuclei), or to provide detailed isotropic composition measurements in the range $Z=1$ through 16 .

\section{Detectors}

The Voyager Cosmic Ray Instrument contains three basic telescope designs, each optimized for a different range of particle energies and types. These telescopes are illustrated schematically in Figure 1. The Voyager instrument consists of two High Energy Telescopes (HET), four Low Energy Telescopes (LET), and a single Electron Telescope (TET). The two HETs, which are double-ended, and each of the four LETs are oriented in different directions to provide unobstructed fields of view and to provide measurements of the cosmic ray anisotropy on a stabilized spacecraft.

Each HET is double-ended; the "A" end entrance aperture is defined by two $150 \mu \mathrm{m}$ thick silicon surface barrier detectors, A1 and A2, while the "B" end aperture is defined by the $2 \mathrm{~mm}$ thick, curved Li drifted detectors, $\mathrm{B} 1$ and B2. These detectors also provide energy loss

\footnotetext{
*NASA/Goddard Space Flight Center, Greenbelt, MD, 20771 **Cal. Inst. of Technology, Pasadena, CA, 91109
}

measurements. The stack of $\mathrm{C}$ detectors in the center consists of seven $3 \mathrm{~mm}$ thick double-grooved Li-drifted detectors connected as four separate central areas and four integral annular guard regions. The four guards, taken together, and either $\mathrm{C} 4$ or $\mathrm{C} 1$ form an anticoincidence cup to identify stopping particles entering from the $A$ end or from the $B$ end, respectively. These two stopping particle trajectories are shown as S1 and S2 in Figure 1.

Particles of higher energy, entering either the $A$ or $B$ end and penetrating the entire $C$ stack, are identified by $\mathrm{B} 1 \cdot \mathrm{B} 2 \cdot \mathrm{C} 1$ coincidences. The trajectory of these particles is labeled $P$. The four guard rings form a cylindrical anticoincidence wall in this mode. No passive collimation is used in HET for any of the three modes of operation.

Table 1 summarizes the characteristics of the HET telescopes and resulting pulse-height measurements. These detector systems are nearly identical to a portion of the ISEE instrument described by von Rosenvinge et al. (Ref. 2).

Each of the four LETs consist of two large-area, thin $\left(2.8 \mathrm{~cm}^{2} \times 35 \mu \mathrm{m}\right.$ ) surface barrier detectors ( $\mathrm{L} 1$ and $\mathrm{L} 2$ ) which define the entrance aperture and provide redyndant measurements of $\mathrm{dE} / \mathrm{dx}$. The $\mathrm{L} 3$ element $\left(4.5 \mathrm{~cm}^{2} \mathrm{x}\right.$ $450 \mu \mathrm{m}$ thick) provides a total residual energy measurement for those particles which do not penetrate and enter the anticoincidence element L4. A lightweight aluminized delrin light baffle and $3 \mu \mathrm{m}$ Al foil window protect the telescope from incident sunlight and associated thermal effects. Table 2 summarizes the characteristics of the LET telescopes and resulting pulse-height measurements.

The Electron Telescope (TET), also shown in Figure 1, consists of a coaxial array of eight double-grooved Li-drifted detectors (D1-D8), and various thicknesses of Tungsten absorber (A1-A6) interspersed between the last six detectors. The entrance aperture is defined by the central areas of the first two detectors (D1 and D2). The outer rings of all eight detectors and the central area of the last detector (D8) form an anticoincidence cup to identify particle events which enter but do not exit the telescope. Double $\mathrm{dE} / \mathrm{dx}$ vs. range analysis, where the range is one of the 5 detectors D3-D7, is employed to determine the incident particle energy. Upper level discriminators in anticoincidence eliminate protons and heavier particles. The geometric factor varies between $1.8 \mathrm{~cm}^{2}$-sr for $\mathrm{D} 1 \cdot \mathrm{D} 2 \cdot \mathrm{D} 3$ events to $0.6 \mathrm{~cm}^{2}-\mathrm{sr}$ for events which penetrate to $\mathrm{D} 7$.

\section{Electronics}

The electronics system to analyze and process particle events from the seven telescopes consists of four major subsystems as illustrated in Figure 2: (1) there are 50 charge-sensitive preamp-shaping amplifier chains, (2) an analog signal processor containing threshold discriminators and coincidence/anticoincidence systems for each telescope and three sets of multi-parameter pulse-height analyzer systems shared between several telescopes, (3) the data subsystems, which include event rate accumulators, a command system and PHA data system, and (4) power converters for detector bias and low voltages. System partitioning takes advantage of the symmetry resulting from grouping a single HET with two LETs, and then duplicating that subsystem for the remaining HET and LETs. This subsystem is referred to as a HET/LET block. Parallel redundancy was the natural 

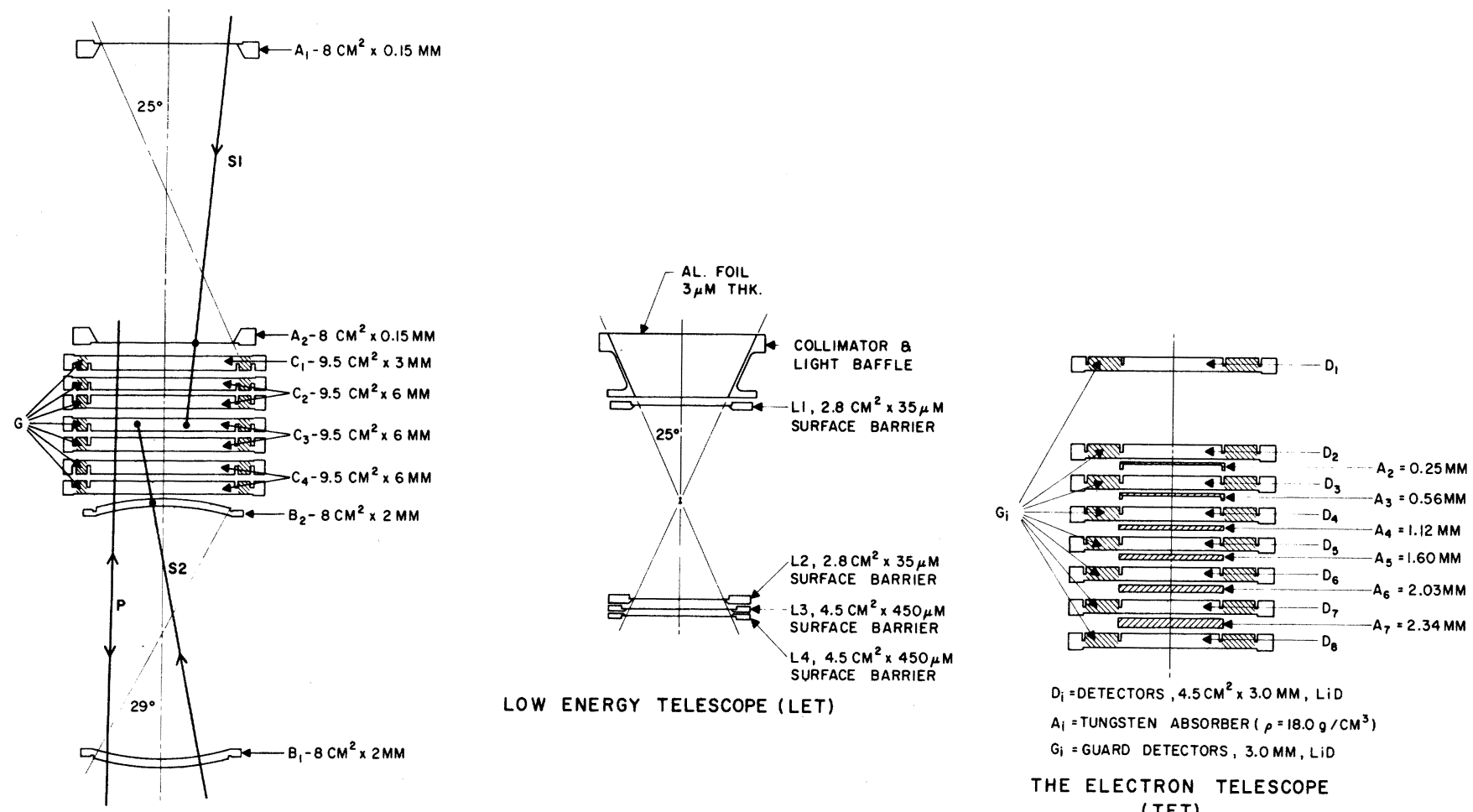

$D_{i}=$ DETECTORS, $4.5 \mathrm{CM}^{2} \times 3.0 \mathrm{MM}$, LiD $A_{1}=$ TUNGSTEN ABSORBER $\left(\rho=18.0 \mathrm{~g} / \mathrm{CM}^{3}\right)$

$G_{i}=$ GUARD DETECTORS, $3.0 \mathrm{MM}$, LID

THE ELECTRON TELESCOPE

(TET)

HIGH ENERGY TELESCOPE (HET)

FIG. 1: Schematic of the HET, LET and TET detector systems.

TABLE 1

HET TELESCOPE PARAMETERS

\begin{tabular}{ccccccc}
$\begin{array}{c}\text { Event } \\
\text { Type }\end{array}$ & $\begin{array}{c}\text { Type of } \\
\text { Analysis }\end{array}$ & $\begin{array}{c}\text { Proton Energy } \\
\text { Range (MeV) }\end{array}$ & $\begin{array}{c}\text { Coincidence } \\
\text { Condition }\end{array}$ & $\begin{array}{c}\text { Detectors } \\
\text { Analyzed }\end{array}$ & $\begin{array}{c}\text { Geometry } \\
\text { Factor } \\
\text { (cm }{ }^{2} \text {-ster) }\end{array}$ \\
\cline { 1 - 2 } $\mathrm{S}_{1}$ & dE/dx vs. $\mathrm{E}$ & $4-57$ & & $\mathrm{~A}_{1} \mathrm{~A}_{2} \overline{\mathrm{C}}_{4} \overline{\mathrm{G}}$ & $\mathrm{A}_{1}, \mathrm{~A}_{2}, \mathrm{C}_{1}+\mathrm{C}_{2}+\mathrm{C}_{3}$ & $1.0-1.7$ \\
$\mathrm{~S}_{2}$ & $\mathrm{dE} / \mathrm{dx}$ vs. $\mathrm{E}$ & $18-70$ & & $\mathrm{~B}_{1} \mathrm{~B}_{2} \bar{C}_{1} \overline{\mathrm{G}}$ & $\mathrm{B}_{1}, \mathrm{~B}_{2}, \mathrm{C}_{2}+\mathrm{C}_{3}+\mathrm{C}_{4}$ & $0.9-1.7$ \\
$\mathrm{P}$ & Triple dE/dx & $70-500$ & & $\mathrm{~B}_{1} \mathrm{~B}_{2} \mathrm{C}_{1}$ & $\mathrm{~B}_{1}, \mathrm{C}_{1}, \mathrm{C}_{2}+\mathrm{C}_{3}+\mathrm{C}_{4}$ & 1.7
\end{tabular}

TABLE 2

LET TELESCOPE PARAMETERS

$(\mathrm{SL}=$ S1ant Condition $)$

\begin{tabular}{|c|c|c|c|c|c|}
\hline Type & Element & $\begin{array}{c}\text { Energy Range } \\
(\mathrm{MeV} / \mathrm{nuc})\end{array}$ & $\begin{array}{c}\text { Coincidence } \\
\text { Condition }\end{array}$ & $\begin{array}{l}\text { Detectors } \\
\text { Analyzed } \\
\end{array}$ & $\begin{array}{l}\text { Geometry } \\
\text { Factor } \\
\left(\mathrm{cm}^{2}-\mathrm{sr}\right) \\
\end{array}$ \\
\hline $\mathrm{s}_{1}$ & $z \leq 2$ & $\mathrm{H}: 3-8.4$ & 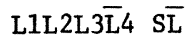 & $\mathrm{L} 1, \mathrm{~L} 2, \mathrm{~L} 3$ & 0.44 \\
\hline $\mathrm{S}_{2}$ & $z \geq 3$ & $\left\{\begin{array}{c}{ }^{16} 0: 5 \cdot 3-17 \\
56 \mathrm{Fe}: 7 \cdot 4-23\end{array}\right\}$ & L1L2L $3 \bar{L} 4 \mathrm{SL}$ & $\mathrm{L} 1, \mathrm{~L} 2, \mathrm{~L} 3$ & 0.44 \\
\hline
\end{tabular}

result; and subsystem design, layout and test were simplified. The TET system, which is somewhat less complicated, was implemented separately. An internal stimulus generator provides test pulses in a predetermined sequence to various preamps.

\section{Preamplifiers:}

A11 preamps utilize two parallel input FETs in a cascade configuration followed by two additional common emitter stages. The input FET current is approximately $3 \mathrm{~mA}$ in both devices, and the preamp noise is $\approx 35 \mathrm{keV}$ fwhm with a simulated detector capacity of $700 \mathrm{pf}$, which corresponds to the A detectors. The pre/post amplifier is shown in Figure 3 in simplified form.

For the HET Telescope, the dynamic range of interest is too wide $(\chi 40,000: 1)$ to be accommodated by the PHA system. To mitigate this burden, these preamplifiers were designed to allow the gain to be toggled by a factor of 5 to 10 . These changes take place in response to a single bit from the experiment command system. An additional feedback network is switched across the preamp by a low-capacity CMOS switch. The preamplifier output is fed to an adjustable-gain 


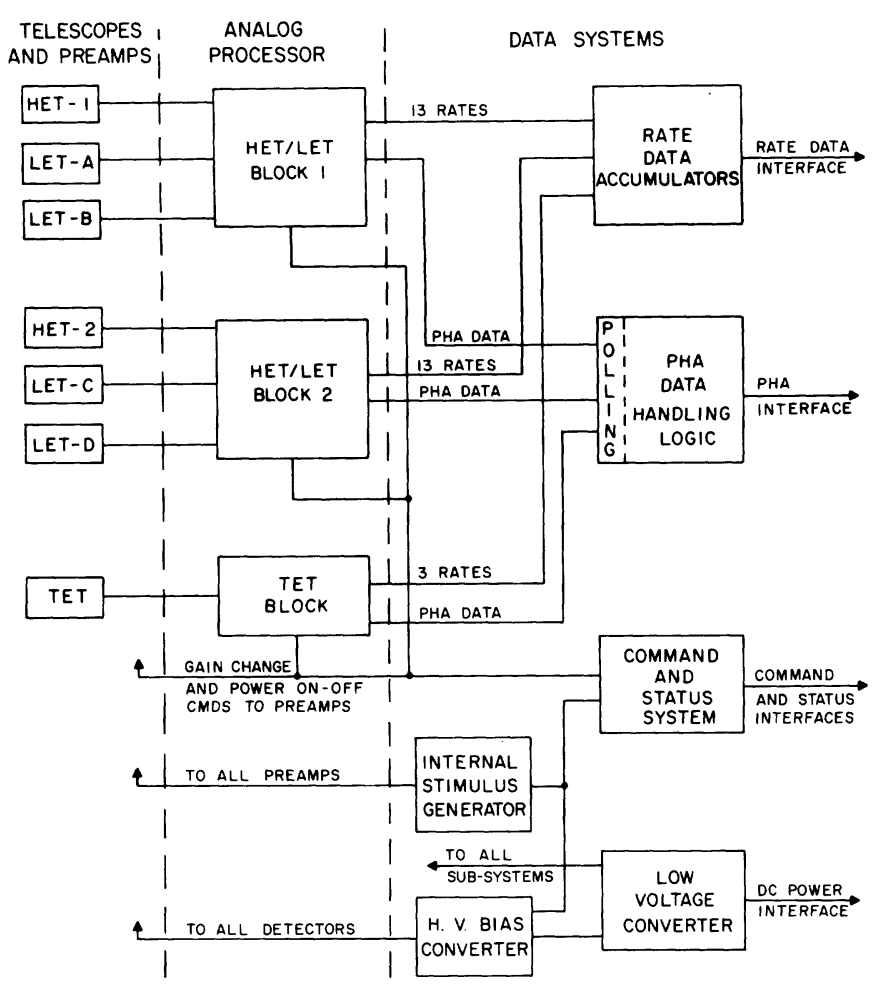

FIG. 2: Simplified Block Diagram of the Voyager Cosmic Ray Instrument. post-amplifier. The post-amp gain is trimmed (to a maximum transfer gain of 10 and full-scale output voltage of $10 \mathrm{~V}$ ) for the desired conversion gain of the PHA system.

The post-amp input network provides pole-zero cancellation to improve the baseline recovery and overload characteristics. The feedback network provides first order Gaussian shaping with a peaking time at approximately $2.2 \mu \mathrm{sec}$. The post-amplifier output provides the low impedance required to drive the cable and the coupling transformer at the input to the PHA electronics. The pre/post-amplifier assembly operates from a $+10 \mathrm{~V}$ and $-6 \mathrm{~V}$ power supplier requiring $\approx 55 \mathrm{mV}$, depending on the detector.

The amplifiers were constructed on PC daughter cards. Sufficient daughters were brought together on a mother board to satisfy the requirements of a particular telescope. The gain adjustment of the post-amp was located on the mother board to allow easy gain matching when the complete system was integrated.

\section{Analog Signal Processor:}

The HET-LET block, shown in detail in Figure 4, supports one HET and two LETs. Two identical such blocks and a separate TET system constitute the analog signal processor. The detectors and associated preamps and shaping amps are shown on the left. Each amplifier output is transformer coupled at the analog system input to provide approximately $55 \mathrm{db}$ common mode noise rejection in the range 0.05 to $1 \mathrm{MHz}$. The linear signals are then connected via buffer amplifiers $(A=60)$ to threshold discriminators, each of which produce a logical "1" output as long as the input signal exceeds the threshold level. The discriminator output pulse width varies between a minimum of $5.5 \mu \mathrm{sec}$ to $\sim 35 \mu \mathrm{sec}$ for a full-scale input. This provides a measure of baseline protection in that a given signal must return nearly to the baseline before that discriminator may be triggered again. This prevents pulse-height analysis of pulses

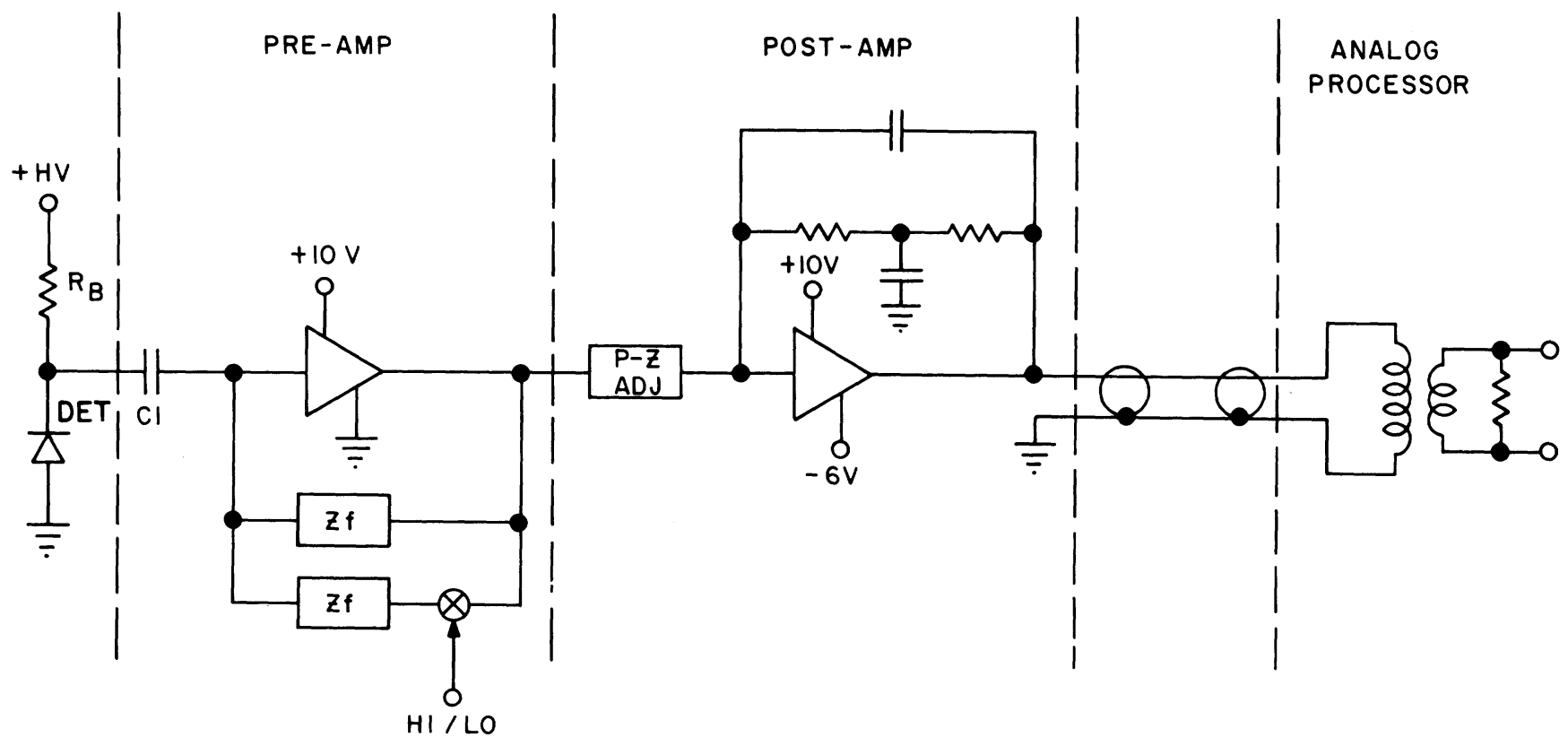

FIG. 3: Diagram of Preamp-postamp. Two Feedback Networks around the Preai: p Provide Hi/Lo Gain Operation. 


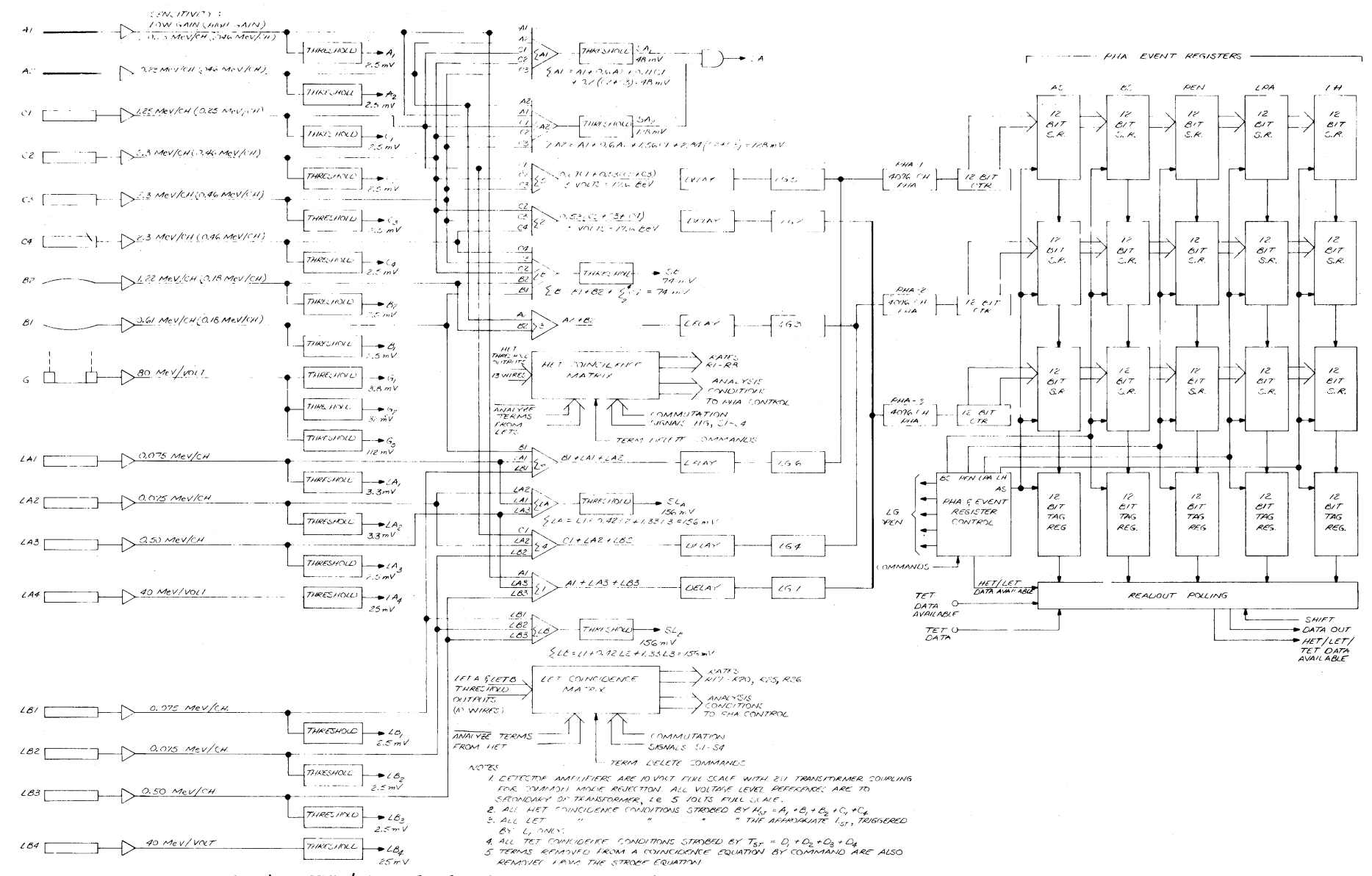

FIG. 4: HET/LET Block Diagram. Two Identical Systems are Used in the Instrument.

piling up on the tail of large preceeding pulses. The threshold level is constant within $+0.7 \%$ over a $40^{\circ} \mathrm{C}$ temperature range.

The individual detector signals are also summed in various combinations by precision linear summing amplifiers prior to pulse-height analysis, and to provide "slant" discriminator terms which identify high-Z particle events separately from low-Z events in the various telescopes. These slant terms are shown in Figure 4 as SA, SB, and SL, where SA is a dual slope term SA1•SA2. The "slant" discriminator is a commonly-used particle separation technique in $\Delta E$ vs. E telescopes.

Figure 5 depicts the energy loss for an A-stopping event in detectors $(\mathrm{C} 1+\mathrm{C} 2+\mathrm{C} 3)$ vs. the energy loss in $(\mathrm{A} 1+0.6 \mathrm{~A} 2)$. The discriminator thresholds SA1 and SA2 plot as straight lines and are positioned between the alpha and lithium particle tracks. Lithium particles will trigger both discriminators, whereas alphas may trigger either separately or neither, depending on their incident energy. Coefficients in the SAl and SA2 equations of Figures 4 and 5 differ because the former is in terms of preamp output voltage and the latter is in terms of energy loss.

Linear signals to be pulse-height analyzed are passed through precision linear summing amplifiers, delay lines, and series-shunt linear gates to the 4096 channel ADCs. The delay line insertion loss is compensated by a buffer amplifier which includes a Robinson base line restorer (Ref. 3) prior to the linear gates. Each ADC input is preceeded by two of the series linear gates and a single shunt gate. Only one of the two series gates is allowed to open, depending on the event type selected for analysis. In some cases, the linear summation is a basic requirement of the measurement; e.g., the total energy in the $\mathrm{C}$ stack of HET $(\Sigma \mathrm{Ci})$. In other cases, the summation is only a means combining signals from various detectors so that a given $\mathrm{ADC}$ can be used for a HET event or for either LET event without the need for additional delay lines and linear gates. In the latter case, the summation mixes desired

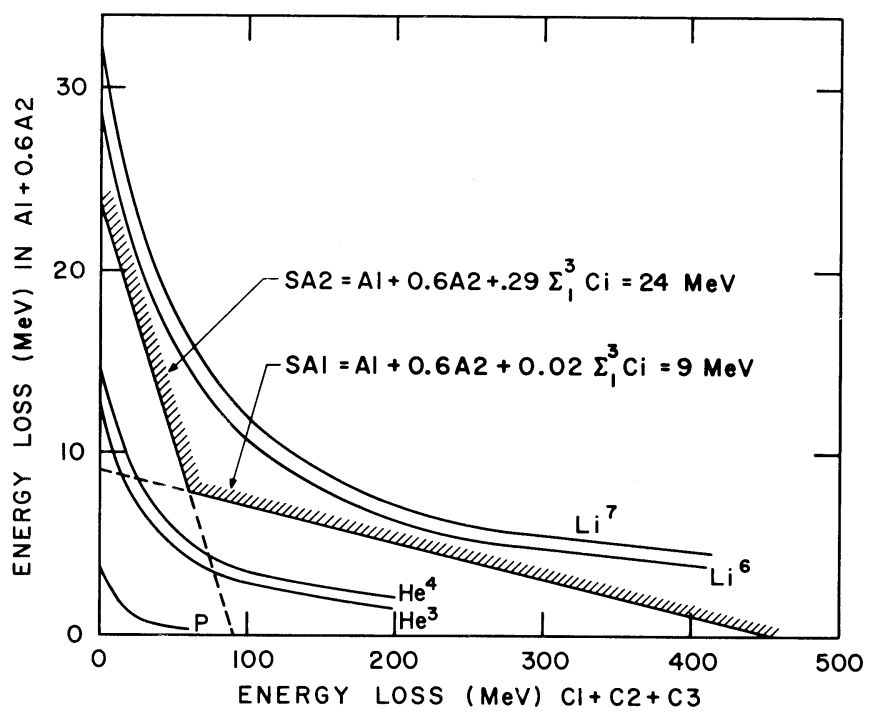

FIG. 5: Energy Loss in A1+0.6A2 vs. Energy Loss in $\Sigma_{1}^{3} \mathrm{Ci}$. Only Lithium and Higher $Z$ Particles Satisfy the Condition SA1-SA2. 
signals from one detector with background noise and possible unwanted pulses from other detectors (e.g., $\sum 1$ output is $A 1+L A 3+L B 3$ and only $A 1$ is of interest for A-stopping events). Hence, all analysis conditions include not only the affirmative terms which define each event type, but also "veto" terms indicating absence of conflicting pulses from unrelated detectors. These veto terms originate from the discriminators which, as discussed earlier, produce an output for at least as long as the input signal exceeds threshold.

The pulse-height analyzer differential non-1inearity is 1 ess than $\pm 1.5 \%$ over the top $99 \%$ of full scale, and retains excellent performance down to threshold (about channe1 2). The PHA conversion gain is not particularly stable $\left(\leqslant 6^{\circ}\right.$ over a $40^{\circ} \mathrm{C}$ range); but this is of little consequence since the instrument is at an essentially constant temperature throughout most of the mission, and because the telescopes are essentially self-calibrating.

Separate 48-bit storage registers for each event type store the three parameter PHA results, along with 12bits of tag information for later readout. The tags uniquely identify each event as to type, origin (telescope ID), slant condition, etc.

Also shown in Figure 3 is a coincidence rate matrix associated with each telescope. All coincidence and PHA event selection logic was implemented using discrete component low-power emitter-coupled logic circuitry, which generates very little noise due to the low signal levels ( -0.6 and 0.9 volts for logic " 1 " and " 0 ", respectively) and constant current consumption. Each matrix contains an array of ECL AND-gates which are strobed on the trailing edge of a delay monostable. Delay time is typically $\gtrsim 2.6$ $\mu \mathrm{sec}$ to accommodate the slowest rise time. Gate input terms are provided by the discriminator outputs; and, for the most part, the presence of any given term (or absence of any given anti-coincidence term) may be deleted by command. There are a total of 29 such gates for the seven telescopes, one corresponding to each rate counter. 1.11 commutation of rate terms is performed at the ECL level prior to the coincidence gates. A total of 45 coincidence conditions are detected, along with 56 singles rates from the various discriminator outputs.

The readout polling system in each of the two HET/LET blocks sequentially scan "data available" flags of their respective event registers, including a TET PHA register not shown in Figure 3 , to optimize the use of available telemetry space. The polling uses an eightposition multiplexer for the six event types, which allows the LET registers to be accessed twice per scan. This system, in conjunction with commutation of high and low gain in the HET preamplifiers, emphasizes analysis and readout of rare events at all times without the use of a rotating priority scheme. A similar, higher level polling system toggles alternately between blocks if both have data available, or selects data from the appropriate block if only one has data.

The TET analog processor is shown in Figure 6 . The charge-sensitive preamps for each of the central areas of D1 through D8, as we11 as the two guards (GA and GB), are identical to the HET and LET designs, but are operated at much reduced power from a lower supply voltage. Both upper and lower level discriminators (UT and LT) are associated with each detector; those for D1 and D2 form a window, W1 and W2, which select electrons and reject protons and heavier particles. Both D1 and D2 signals are digitized when the coincidence condition $W 1 W 2 D 3 \bar{D} 8 G$ is satisfied and the storage registers are available. The status of all remaining UT's and LT's are stored in the tag register to provide range information.

The analog processor was fabricated with a combination of printed circuit and thick film ceramic daughter modules approximately $1.5 \mathrm{~cm}$ high and $3.8 \mathrm{~cm}$ or more in length. Dual transistors in TO-89 packs and other low-profile parts are used throughout to permit the modules to be mounted on $2.54 \mathrm{~mm}$ centers. Approximately 500 such modules, and other discrete parts, such as delay lines, are mounted on five $15.7 \times 20.1 \mathrm{~cm}$ mother boards. All trim components are mounted on the mother boards for ease of adjustment after assembly.

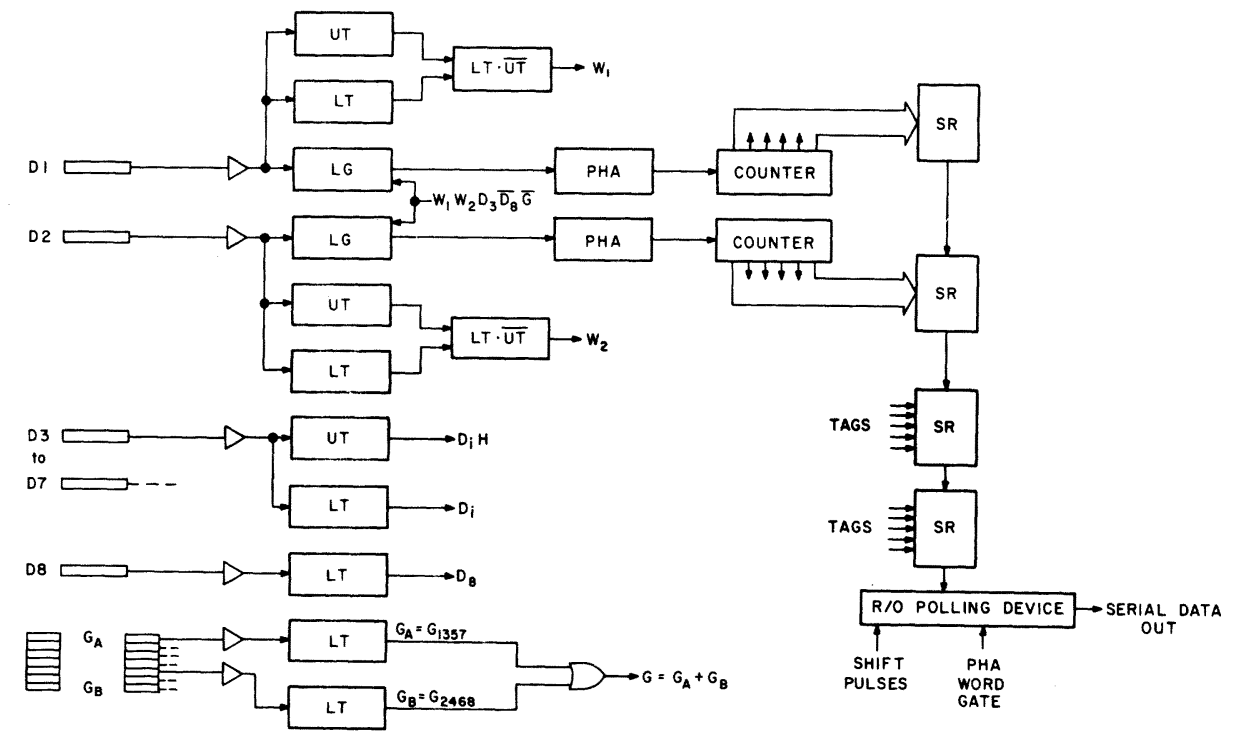

$\begin{array}{ll}\text { UT }=\text { UPPER } & \text { THRESHOLD } \\ \text { LT }=\text { LOWER } & \text { THRESHOLD }\end{array}$

FIG. 6: TET Block Diagram 
Rate Counters and Command System:

The various rate outputs from the coincidence matrices are counted in custom PMOS LSI quasi-logarithmic counters developed at GSFC (Ref. 4). A single chip contains a 24-bit binary counter, a quasi-log compressor which converts the 24-bit counter data to a 12-bit output, and a readout storage register. There are 30 such counters in the data system.

The command system accepts 12 -bit. serial commands from the spacecraft which are formatted as a 4-bit address and 8-bit data field. Of the 128 possible bits thus available, 95 -are used to delete terms from the coincidence equations, disable analysis of specific event types, remove power from any of the preamps, control the high/low gain switching of HET preamps, and control other experiment functions. A11 95 bits, as well as other indicator bits, are read out in the engineering status data. Command and status data is distributed within the experiment in 8-bit serial form to reduce inter-subsystem wiring.

Two additional pulse commands are used to turn on the bias converter and to initiate an internal stimulus cycle which applies fixed amplitude test pulses to various preamps to verify experiment operation.
Approximately 240 CMOS integrated circuits on three multilayer boards make up these systems. The CMOS and PMOS are housed within a separate container of $0.6 \mathrm{~mm}$ thick aluminum to provide protection against radiation damage during Jupiter fly-by.

\section{Mechanical, Radiation and Thermal} Considerations

An overall view of the instrument is shown in Figure 7.

All the electronics, except preamplifiers, are mounted in a $20.3 \times 28.6 \times 12.7 \mathrm{~cm}$ high volume with thin honeycomb top and bottom plates. The preamps are enclosed within a separate cover in an additional $5 \mathrm{~cm}$ high volume above the upper honeycomb plate. Al1 telescope are mounted, porcupine fashion, on the upper honeycomb plate. Much of the structural support between the two honeycomb plates is provided by the electronics, particularly by the data system because of its additional housing for radiation protection. The side walls are $0.6 \mathrm{~mm}$ aluminum.

The long thin strips visible on the upper surface of the preamp cover may be removed to provide access to the preamp test imput and output monitor connectors. These are push-on style minature coaxial connectors mounted directly on the preamp mother board.

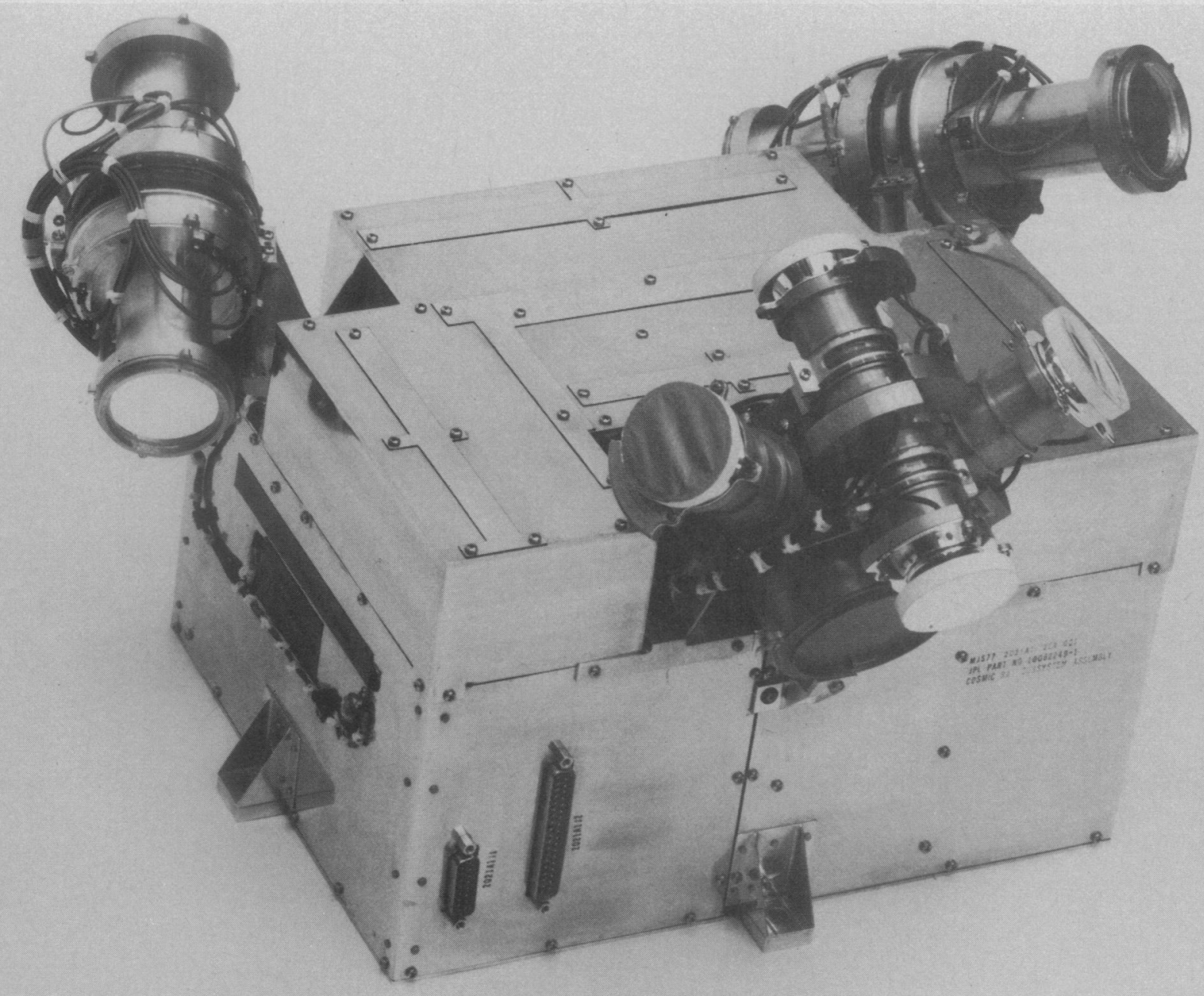

FIG. 7: The Voyager Cosmic Ray Instrument 
Al1 radiation damage-sensitive components (primarily CMOS) were mounted as far from the exterior as possible; those not shielded by at least $.7 \mathrm{gm} / \mathrm{cm}^{2}$ equivalent were spot-shielded with 10 -mil Ta foil.

A thermal blanket enclosed the entire instrument, except for the LET telescope apertures and an area on the bottom which was fitted with louvers for thermal control. Additional heaters were mounted on each end of the electronics box, as seen in Figure 7 .

Temperature of the Voyager 1 instrument throughout the mission has varied between $+20^{\circ} \mathrm{C}$ shortly after launch to $+3^{\circ} \mathrm{C}$ with 3.7 watts of supplemental heater power.

\section{Performance}

Figure 8 is an example of pulse-height spectra obtained from HET-2 A-stopping events in the low gain mode during the first seven months of operation. Shown on the vertical and horizontal axis are channel numbers of the A1 and $A 2$ pulse heights resulting from A1A2SAC $4 \bar{G}$ events. The data is compressed to 8 to 1 ; i.e., each row and column represents the total events in a group of eight consecutive PHA addresses.

The effect of the SA discriminator is apparent near the origin; the Al intercept is about 100 channels and the A2 intercept is about 170 channels for events for which there is no energy loss in the $C$ detectors. The carbon, nitrogen and oxygen distributions are readily apparent; and the neon, magnesium and part of the silicon line are shown. Figure 8 depicts only the bottom one-fourth of the A1 and A2 pulseheight analyzer dyhamic range; hence the highest $\mathrm{Z}$ particle tracks are not included. The endpoints of each track are determined by detector thickness and electronic conversion gain; hence any temperature drift in the electronics is readily apparent.

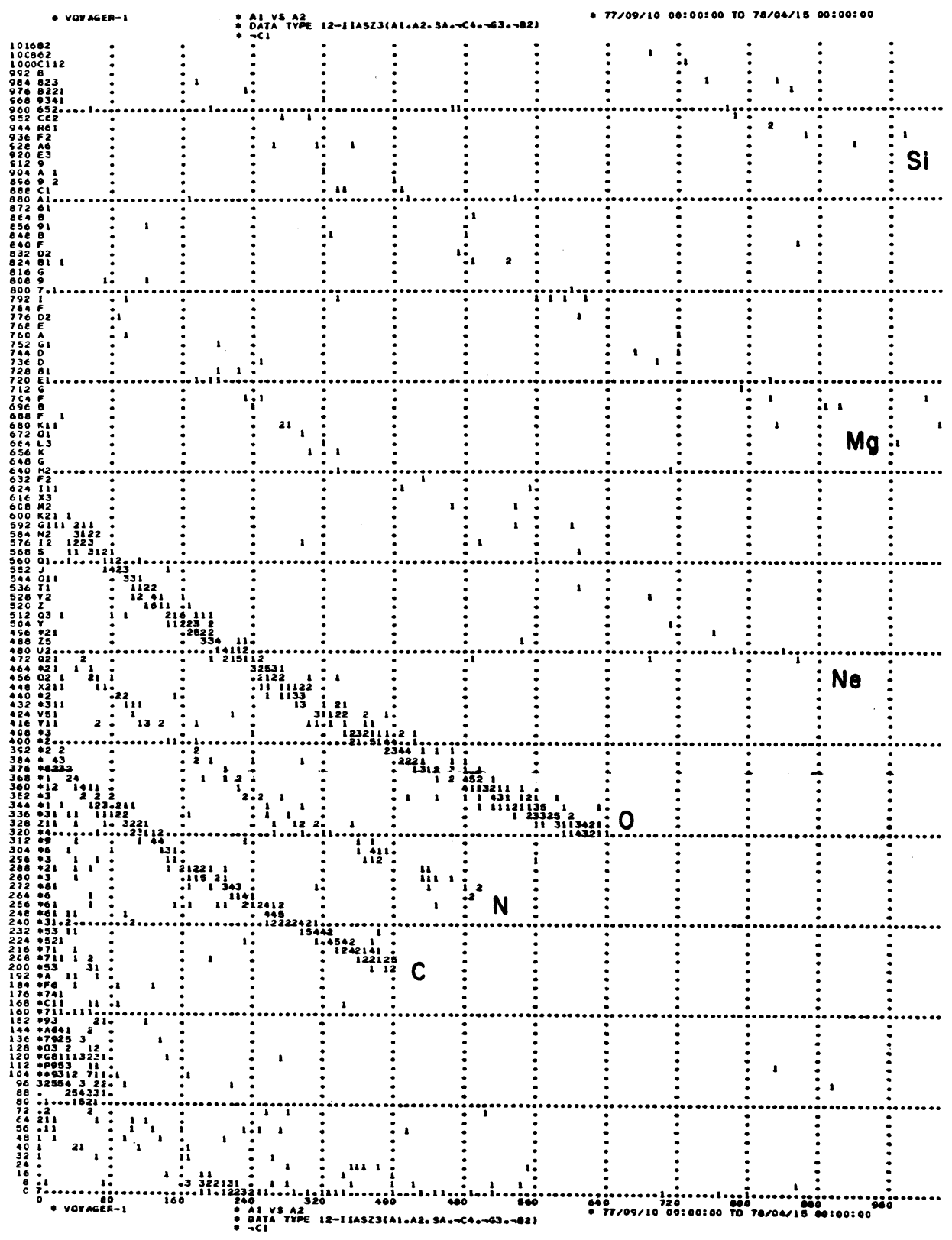

FIG. 8: Distribution in A1 vs. A2 space of PHA data for A-stopping events in one HET. All low-gain data for the period Sept. 177 through mid-April 178 is shown. Numbers indicate number of occurrences of specified A1 and A2 addresses. 
The development of the Voyager Cosmic Ray Experiment was a joint effort of the California Institute of Technology, the University of New Hampshire, and NASA/Goddard Space Flight Center. The authors wish to thank F. Yates and B. Gauld of Caltech (GSE hardware and sof tware development), and D. Argo and N. Lal of the Computer Sciences Corporation at GSFC for pre- and post-launch sof tware development. The LET and TET designs, testing and calibrations at Caltech were capably performed by $S$. Hartman and $R$. Cook, respectively. The analog signal processor was designed and tested by Spacetac, Inc., Burlington, MA--we particularly acknowledge the efforts of G. Adaniya, A. Rizzo, J. Zukauskis, G. Pacquin, J. Bernstein and N. E1liot. Surface barrier detectors were obtained from Ortec, Inc., and the Li-drifted detectors were supplied by the Kevex Corp. At GSFC, the preamps, data systems and overall experiment assembly was performed by M. Beazley, M. Powers and S. Allison; the rate counting system was provided by $\mathrm{H}$. White; and mechanical design of the telescopes and main structure was provided by $\mathrm{H}$. Trexel and

F. Shaffer. G. Evans and J. Mills were particularly patient and helpful in the preparation of this manuscript. T. Conlon kindly provided the data shown in Figure 8 .

We also wish to acknowledge the patience and support of the Voyager Project office at JPL, particularly $\mathrm{J}$. Otte and E. Franzgrote, and the many individuals of the integration and test crews. To all, we extend our sincere thanks for their tireless and successful efforts.
(1) E.C. Stone, R.E. Vogt, F.B. McDonald, B.J. Teegarden, J.H. Trainor, J.R. Jokipii and W.R. Webber, "Cosmic Ray Investigations for the Voyager Missions; Energetic Particle Studies in the Outer Heliosphere--and Beyond," Space Science Reviews 21, No. 3, 355, 1977.

(2) T.T. von Rosenvinge, F.B. McDonald, J.H. Trainor, M.A.I. Van Hollebeke and L.A. Fisk, "The Medium Energy Cosmic Ray Experiment for ISEE-C," Geoscience Electronics GE-16, No. 3, 208, 1978.

(3) L.B. Robinson, "Reduction of Baseline Shift in Pulse-Amplitude Measurements," Rev. Sci. Instr. 32, 1057, 1961.

(4) H.D. White, Jr., and D.C. Lokerson, "The Evolution of IMP Spacecraft MOSFET Data Systems," IEEE Trans. Nuc. Sci. NS-18, No. 1, 233, 1971. 